\title{
Alcohol Use Disorder Identification Test (AUDIT): explorando seus parâmetros psicométricos
}

\author{
Alcohol Use Disorder Identification Test (AUDIT): \\ exploring its psychometric parameters \\ Walberto Silva dos Santos', Valdiney Veloso Gouveia2, Darlene Pinho Fernandes', \\ Sarah Stella Bomfim de Souza', Alex Sandro de Moura Grangeiro'
}

\section{RESUMO}

Objetivo: $O$ presente estudo teve como objetivo conhecer evidências de validade e precisão do Alcohol Use Disorder Identification Test (AUDIT). Métodos: Contou-se com uma amostra de conveniência (não probabilística) de 547 estudantes universitários de Fortaleza (CE), com idade média de 21,6 anos ( $d p=4,86$; amplitude de 18 a 53 ), a maioria do sexo masculino $(51,5 \%)$, solteira $(91,4 \%)$ e católica $(62,5 \%)$. Os participantes responderam ao AUDIT e a perguntas demográficas. Procurando conhecer a estrutura fatorial, além de estatísticas descritivas, realizou-se uma Análise de Componentes Principais. Adicionalmente, a fim de avaliar a precisão do instrumento, efetuaram-se cálculos de alfa de Cronbach (consistência interna), correlações de $r$ de Pearson e coeficiente de correlação intraclasse - ICC (precisão teste-reteste). Resultados: De acordo com a análise de componentes principais com rotação oblimin, a estrutura bifatorial do AUDIT mostrou-se coerente, com todos os itens apresentando saturações satisfatórias, superior a |0,40|, tendo o Fator 1 explicado 47,5\% da variância total com alfa de 0,84 e o Fator 2 explicado 11,6\% da variância total com alfa de 0,69. Os resultados do teste-reteste indicaram correlação forte entre os dados obtidos na primeira $\left(\mathrm{t}_{1}\right)$ e segunda $\left(t_{2}\right)$ aplicação $\left(r_{t t}=0,94, p<0,01\right)$, sem diferença significativa de médias nos dois tempos $\left(m_{t 1}\right.$ $\left.=0,37, d p=0,49 ; m_{t 2}=0,34, d p_{2}=0,47 ; p>0,05\right)$, com ICC satisfatório (0,96). Conclusões: Os achados apoiaram a adequação psicométrica do AUDIT, com as análises fatoriais exploratórias apontando como mais satisfatória a estrutura com dois fatores, bem como atestaram sua boa estabilidade temporal.

\section{ABSTRACT}

Objective: This study aimed to know evidences of validity and reliability of the Alcohol Use Disorder Identification Test (AUDIT). Methods: For this purpose, we considered 547 college students from Fortaleza (CE) with a mean age of 21.6 years ( $s d=4.86$, varying from 18 to 53), mostly male (51.5\%), single (91.4\%) and catholic (62.5\%). The participants answered the AUDIT and demographic questions. Looking to know AUDIT's factorial structure, we carried out a principal component analysis. Additionally, in order to evaluate its reliability, we calculated Cronbach's alpha (internal consistency), Pearson correlation $r$ and intraclass coefficient

1 Universidade Federal do Ceará (UFC).

2 Universidade Federal da Paraíba (UFPB).

Recebido em

$7 / 5 / 2012$

Aprovado em

$6 / 8 / 2012$

Endereço para correspondência: Walberto Silva dos Santos Departamento de Psicologia, Centro de Humanidade, Área II Av. da Universidade, 2762, Benfica 60020-180 - Fortaleza, CE Telefone: (85) 3366-7723 


\section{Keywords}

AUDIT, alcoholism, validation studies, screening instrument, parameters. correlation - ICC (test-retest). Also, descriptive statistics were evaluated. Results: According to the principal component analysis using oblimin rotation, the bifactorial structure of the AUDIT proved to be consistent, once all items showed satisfactory saturations exceeding $|0.40|$, with the first factor explaining $47.5 \%$ of the total variance with alpha of 0.84 and Factor 2 explaining $11.6 \%$ of the total variance with alpha of 0.69 . The results of the test-retest showed strong correlation between data obtained in the first $\left(\mathrm{t}_{1}\right)$ and in the second $\left(\mathrm{t}_{2}\right)$ applications $\left(r_{t t}=0.94, p<0.01\right)$, with no significant difference between the means in both applications ( $m_{t 1}=0.37, s d=0.49,=0.34 m_{t t^{\prime}} d p_{2}=0.47, p>0.05$ ) with satisfactory ICC (0.96). Conclusions: The findings support the psychometric adequacy of the AUDIT, with the exploratory factor analysis pointing as more satisfactory the structure with two factors, and also showing its good temporal stability.

\section{INTRODUÇÃO}

O álcool tem sido considerado o psicotrópico mais utilizado em todo o mundo'. Seu consumo, apesar de lícito e aceito na maioria dos países, aparece relacionado com um conjunto de problemas de ordem individual e social, por exemplo, índices de acidentes de trânsito, homicídios, suicídios, agressões, doenças cardiovasculares e outras patologias graves². Nesses contextos, aponta-se que os riscos associados ao consumo abusivo de álcool não surgem aleatoriamente, mas estão ligados e são potencializados pelo padrão de consumo dessa substância ${ }^{3}$.

No intuito de possibilitar o monitoramento e a vigilância desses padrões de ingestão, pesquisadores vêm estudando e desenvolvendo instrumentos que buscam auxiliar na triagem de usuários na população. Apesar disso, uma busca nas bases de dados Index Psi ${ }^{4}$ (2011) e Google Acadêmico (2011), usando como entrada os termos "álcool", "consumo", "consumo abusivo", "consumo nocivo", "escala", "questionário", "instrumento" e "medida" e limitando a busca a partir do ano 2001, permitiu verificar que, no Brasil, apesar do número expressivo de publicações que identificam e relacionam diversos problemas de saúde com o uso abusivo de álcool, ainda são escassos os estudos cujos objetivos envolvem a construção e/ou a adaptação de medidas voltadas para a detecção do uso nocivo de bebidas alcoólicas em grandes amostras (medidas de screen).

Dentre as diversas medidas utilizadas para detecção do consumo de álcool em seus distintos padrões, destaca-se o Alcohol Use Disorder Identification Test (AUDIT), instrumento originalmente desenvolvido pela Organização Mundial de Saúde (OMS) no fim da década de 1980, a partir de um projeto que envolveu seis países (Austrália, Bulgária, Quênia, México, Noruega e Estados Unidos) ${ }^{6}$. Esse instrumento se configura, na atualidade, como uma das medidas mais empregadas em todo o mundo para a identificação de grupos de risco e rastreamento do uso inadequado de álcool em amostras clínicas e da população geral ${ }^{7,8}$.

Não obstante a ampla utilização do AUDIT, na literatura da área ainda não há consenso acerca de sua dimensiona- lidade. Publicações nessa direção têm indicado estruturas compostas por um, dois e três fatores ${ }^{6,7}$. De acordo com Rist et al. ${ }^{8}$, os estudos em que se observa uma estrutura unifatorial são raros, ao passo que os resultados encontrados para modelos que envolvem estruturas com dois e três fatores são mais frequentes. Além disso, como apontam Meneses-Gaya et al. ${ }^{7}$, dependendo da amostra, as análises fatoriais efetuadas com matrizes de dados provenientes do AUDIT podem produzir resultados diferentes. Se, por um lado, em amostras procedentes de populações com alta prevalência de dependência os resultados corroboram o modelo unifatorial, por outro, naquelas em que se observa baixa prevalência de transtornos relacionados com o consumo de álcool, os resultados remetem à estrutura composta por dois ou três fatores ${ }^{9}$.

Quanto à fidedignidade, na maioria dos estudos, o AUDIT apresenta alfas de Cronbach iguais ou superiores a $0,80^{7}$. Além disso, ainda é possível localizar publicações com relação à sua precisão teste-reteste. Selin ${ }^{10}$, por exemplo, em um estudo que contou com 457 pessoas da população geral, observou, em intervalo de 30 dias entre as aplicações, uma correlação de $0,84(p<0,001)$ para a pontuação total do AUDIT.

De fato, desde a sua elaboração, o AUDIT tem sido expressivamente empregado nos estudos acerca do consumo de álcool com amostras clínicas, da população geral, de estudantes dos ensinos médio e superior ${ }^{11}$, sendo, inclusive, recomendado pelo Ministério da Saúde para levantamentos na atenção básica ${ }^{12}$. Apesar disso, como apontam Meneses-Gaya et al. ${ }^{7}$, um número pequeno de pesquisas tem analisado aspectos importantes sobre suas qualidades psicométricas, como validade, fidedignidade e estrutura fatorial, sobretudo em países com idiomas diferentes do inglês, o que compromete a comparação dos achados entre estudos.

No Brasil, por exemplo, ainda são poucas as pesquisas que analisam as suas propriedades psicométricas 9,13,14. Considerando os últimos 10 anos, localizou-se apenas um estudo em que se oferecem mais detalhes sobre sua adaptação ao contexto brasileiro, sendo os seus resultados provenientes da cidade de Salvador ${ }^{9}$. Nos demais estudos, os únicos parâmetros comumente disponibilizados têm sido a consistência 
interna (alfa de Cronbach) ${ }^{13}$ e a validade de critério (análise da curva Receiving Operator Characteristic - ROC) ${ }^{14}$, que, por si só, não podem ser avaliados como indicadores de validade fatorial do instrumento ${ }^{15}$. Tal constatação se torna ainda mais relevante quando se avalia, no campo da saúde pública, a necessidade cada vez mais proeminente de contar com medidas curtas, de fácil aplicação e que reúnam parâmetros satisfatórios de validade e precisão. Tais aspectos justificaram a realização do presente estudo, cujo objetivo principal foi avaliar a qualidade psicométrica do AUDIT. Especificamente, pretendeu-se verificar seus parâmetros de validade fatorial e precisão, observando, neste último caso, a consistência interna e a estabilidade temporal (precisão teste-reteste).

\section{MÉTODO}

Contou-se com a participação de 547 estudantes universitários da cidade de Fortaleza (CE), com idade média de 21,6 anos ( $d p=4,86$; amplitude de 18 a 53), a maioria do sexo masculino $(51,5 \%)$, solteira $(91,4 \%)$, residindo com os pais $(79,7 \%)$ e da religião católica (62,5\%). Desse total, após aproximadamente 45 dias, voltaram a participar do estudo (reteste) 49 estudantes com idades variando entre 18 e 29 anos ( $m$ $=20,0 ; d p=2,03)$, a maioria do sexo feminino $(63,3 \%)$ e da religião católica (65,3\%). A amostra foi de conveniência (não probabilística), participando aquelas pessoas que, ao serem convidadas, concordaram em colaborar com a pesquisa. Este estudo foi aprovado pelo Comitê de Ética em Pesquisa da Universidade Federal do Ceará (Protocolo no 87/10). Os dados foram coletados após o participante ter aceitado e assinado o Termo de Consentimento Livre e Esclarecido.

Os participantes responderam a questões constantes em um livreto composto por seis partes. No entanto, em razão dos objetivos do presente estudo, apenas duas são consideradas:

Alcohol Use Disorder Identification Test (AUDIT) ${ }^{6,16}$. Esta medida é composta por 10 itens que abrangem três domínios teóricos: 1) Frequência do consumo de álcool (por exemplo, "Com que frequência você consome alguma bebida alcoólica?"), 2) dependência do consumo de álcool (por exemplo, "Com que frequência você achou que não seria capaz de controlar a quantidade de bebida depois de começar?") e 3) consequências negativas do consumo de álcool (por exemplo, "Com que frequência você sentiu culpa ou remorso depois de beber?"). Inicialmente, o participante é convidado a pensar sobre seu consumo de bebidas alcoólicas nos últimos 12 meses, respondendo aos itens por meio de diferentes escalas de resposta. O primeiro item envolve a frequência do consumo e é respondido em uma escala de cinco pontos, variando entre 0 (Nunca) e 4 (Quatro ou mais vezes por semana). O item 2, que mensura a quantidade de bebida alcoólica consumida, inclui cinco opções de respostas que se distribuem entre as opções: não bebo (0); 1 ou 2 "doses" (1); 3 ou 4 "doses" (2);
5 ou 6 "doses" (3); 7 a 9 "doses" (4); e 10 ou mais "doses" (5). Para os itens 3, 4, 5, 6, 7 e 8 as respostas podem variar entre os seguintes extremos: Nunca (0) e Todos os dias ou quase todos (4). As questões 9 e 10 apresentam três alternativas de resposta, a saber: Não (0); Sim, mas não no último ano (2); e Sim, durante o último ano (4). Para o cálculo da pontuação total, somam-se os valores referentes a cada resposta, sendo 40 a pontuação máxima da escala.

A última parte, denominada Caracterização da Amostra, consta de perguntas como sexo, escolaridade e religião. Especificamente, perguntou-se com que frequência essa experiência ocorreu ou tem ocorrido, sendo a pergunta respondida em uma escala de sete pontos, variando de 0 (nunca) a 6 (todos os dias).

Para o contexto brasileiro, a versão em português do AUDIT foi desenvolvida por Méndez ${ }^{14}$ e, posteriormente, adaptada por Lima et al. ${ }^{9}$. Para o presente estudo, optou-se por utilizar a versão proposta por Lima. Contudo, antes de proceder à coleta dos dados, com o objetivo de verificar a adequabilidade dessa versão do AUDIT à população-meta, o instrumento foi submetido à validação semântica, nesta etapa, contando-se com um grupo de 10 indivíduos (juízes), escolhidos por conveniência na população-meta do estu$\mathrm{do}^{15}$. Estes avaliaram os itens e as instruções do instrumento, indicando se estavam inteligíveis. Com base nos resultados dessa avaliação, algumas modificações foram realizadas: 1) Alteração da opção de resposta para os itens 3 a 8, em que a expressão "uma vez por mês ou menos" foi substituída por "menos de uma vez por mês"; 2) Inclusão da opção "não bebo" na questão dois; e 3) Exclusão da expressão "durante o último ano" das questões 4 a 8, uma vez que as instruções já levavam o participante a pensar em seu consumo, considerando esse período.

Após a redação final e impressão dos instrumentos, um grupo de três colaboradores, devidamente treinados, foi encarregado de aplicar os questionários. A aplicação foi realizada coletivamente nos ambientes das universidades, em horário cedido pelos dirigentes e professores. Na ocasião, assegurou-se aos participantes o caráter confidencial de suas respostas e os aplicadores indicaram um endereço onde eles poderiam obter informações sobre os resultados finais do estudo. O tempo médio de resposta foi de 15 minutos e, após a coleta dos dados, excluíram-se da amostra aqueles questionários em que os respondentes deixaram mais de 10\% dos itens de uma das medidas sem resposta ou responderam de forma diferente ou utilizando escala distinta das propostas nos questionários.

As análises foram efetuadas por meio do software SPSS (versão 19). Além das estatísticas descritivas (tendência central e dispersão), realizaram-se, inicialmente, um teste $t$ de Student, para avaliar o poder discriminativo dos itens, comparando suas pontuações médias em função de grupos-critério internos, e correlações item-total corrigidas, para avaliar 
a homogeneidade deles. Em seguida, verificou-se a adequação da matriz de dados correspondente com o fim de se realizar uma análise fatorial exploratória, considerando o Kaiser-Meyer-Olkim (KMO), que precisa ser de pelo menos 0,60 para suportar esse tipo de análise, e o Teste de Esfericidade de Bartlett, cujo valor do qui-quadrado deve ser estatisticamente significativo ${ }^{17}$. Para conhecer a estrutura fatorial do conjunto de itens, foi realizada uma Análise de Componentes Principais. Salienta-se que, para estabelecer o número de fatores a ser retido, foram considerados os critérios de Kaiser, Cattel e Horn (Análise Paralela). Além disso, a fim de conhecer a confiabilidade do instrumento, efetuou-se o cálculo do alfa de Cronbach, bem como dos coeficientes de correlações $r$ de Pearson e coeficiente de correlação intraclasse (ICC) para observar a estabilidade teste-reteste.

\section{RESULTADOS}

Inicialmente, procurou-se avaliar o poder discriminativo dos itens. Nessa análise, foram estabelecidos grupos-critérios internos, considerando os participantes cujas pontuações totais se localizaram abaixo e acima da mediana empírica (Md = 5) das pontuações apresentadas pelos respondentes no AUDIT, chamando-os, respectivamente, de grupo-critério inferior e superior. Mediante o uso do teste $t$, foram comparadas as médias dos dois grupos para cada um dos itens, com o objetivo de verificar se conseguiam diferenciar os respondentes com pontuações próximas. Em seguida, efetuaram-se os cálculos das correlações item-total corrigidas e, finalmente, do alfa de Cronbach do conjunto total, observando seus valores em função da exclusão dos itens com baixa homogeneidade. Os resultados dessas análises são resumidos na tabela 1.
De acordo com tabela 1, todos os itens do AUDIT conseguiram diferenciar os grupos-critério $(p<0,001)$, mesmo quando esses apresentaram pontuações próximas. Quanto à homogeneidade, as correlações $\left(r_{i t}\right)$ variaram entre 0,41 (Alguma vez na vida algum parente, amigo, médico ou outro profissional da saúde já se preocupou com você por causa de bebida ou Ihe disse para parar de beber?) e 0,79 (Com que frequência você toma "seis ou mais doses" em uma ocasião?), porém todas acima do mínimo aceitável $(0,20)^{18}$. Quanto à consistência interna para o conjunto total de itens, o alfa de Cronbach foi de 0,86 , valor que cumpre o ponto de corte estabelecido na literatura $(0,70)^{19}$.

Após essas análises, para atender aos objetivos propostos, procurou-se conhecer as possíveis estruturas fatoriais para o AUDIT. No entanto, antes de prosseguir com as análises fatoriais exploratórias, verificou-se a fatorabilidade da matriz, pois, caso os itens (variáveis) não apresentem variância em comum, a análise fatorial não é possível. Neste estudo, a fatorabilidade da matriz de dados foi confirmada por meio do $K M O=0,88$ e do Teste de Esfericidade de Bartlett, $X^{2}$ $(45)=2497,58 ; p<0,001^{17}$. Uma vez que os resultados foram favoráveis à realização dessa análise, tornou-se imprescindível estabelecer quantos fatores podem ser extraídos da matriz de dados, sobretudo porque a sub ou superextração de componentes pode distorcer os resultados de análises posteriores ${ }^{20}$. Para a identificação desse número, diferentes critérios podem ser utilizados ${ }^{21}$, entretanto, neste estudo, três são considerados: Kaiser, Cattell e Horn.

Para averiguar o número de fatores a extrair com base no que propõe Kaiser, inicialmente foi efetuada uma análise de componentes principais, sem fixar o número de fatores e o tipo de rotação. Nessa análise, foi possível encontrar até dois componentes que atenderam ao critério de valor próprio (eigenvalue) igual ou superior a um. Eles explicaram conjun-

Tabela 1. Poder discriminativo, correlação item-total e precisão dos itens do AUDIT

\begin{tabular}{|c|c|c|c|c|c|c|c|}
\hline \multirow{2}{*}{ Item } & \multicolumn{2}{|c|}{ Grupo Inferior } & \multicolumn{2}{|c|}{ Grupo Superior } & \multirow{2}{*}{$t$} & \multirow{2}{*}{$r_{i . t}^{b}$} & \multirow{2}{*}{ Precisão do item } \\
\hline & m & $d p$ & m & $d p$ & & & \\
\hline \multicolumn{8}{|l|}{ Fator $(\alpha=0,86)$} \\
\hline 02 & 0,45 & 0,66 & 3,01 & 1,14 & $30,85^{\mathrm{a}}$ & 0,72 & 0,83 \\
\hline 01 & 0,39 & 0,66 & 1,95 & 1,14 & $24,86^{\mathrm{a}}$ & 0,76 & 0,83 \\
\hline 03 & 0,06 & 0,24 & 1,60 & 0,96 & $24,44^{\mathrm{a}}$ & 0,79 & 0,82 \\
\hline 08 & 0,03 & 0,16 & 1,00 & 0,96 & $15,86^{\mathrm{a}}$ & 0,68 & 0,84 \\
\hline 07 & 0,04 & 0,20 & 0,82 & 0,94 & $12,80^{\mathrm{a}}$ & 0,53 & 0,85 \\
\hline 09 & 0,04 & 0,27 & 1,31 & 1,56 & $12,68^{\mathrm{a}}$ & 0,46 & 0,86 \\
\hline 04 & 0,04 & 0,36 & 0,81 & 1,12 & $10,39^{\mathrm{a}}$ & 0,58 & 0,84 \\
\hline 10 & 0,01 & 0,12 & 0,87 & 1,50 & $9,04^{\mathrm{a}}$ & 0,41 & 0,86 \\
\hline 05 & 0,00 & 0,00 & 0,42 & 0,84 & $7,99^{\mathrm{a}}$ & 0,53 & 0,85 \\
\hline 06 & 0,00 & 0,00 & 0,19 & 0,68 & $4,47^{\mathrm{a}}$ & 0,42 & 0,86 \\
\hline
\end{tabular}

${ }^{\mathrm{a}} p<0,001 ;{ }^{\mathrm{b}} r_{i f}=$ correlação item-total corrigida. 
tamente 59,1\% da variância total. Contudo, uma vez que tal critério superestima o número de componentes a extrair, decidiu-se ter em conta outros dois critérios mais robustos ${ }^{22}$ : a distribuição gráfica dos valores próprios (Cattell) e a geração aleatória de tais valores (Horn ou Análise Paralela). Com base no critério de Cattell (screeplot), é possível identificar apenas um fator, o que pode ser observado na figura 1.

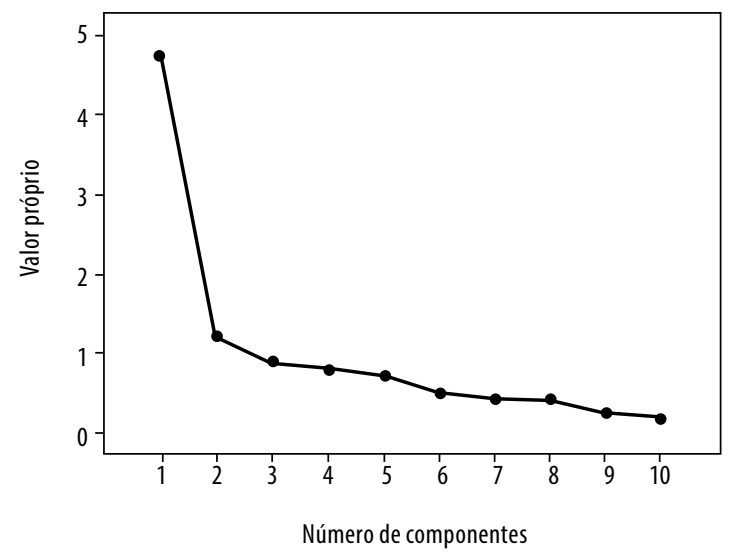

Figura 1. Representação gráfica dos valores próprios (critério de Cattell).

A análise paralela (critério de Horn) contrasta os eigenvalues (valores próprios) gerados pela matriz de componentes (observados) com aqueles produzidos aleatoriamente (simulados), em função do tamanho da amostra e do número de itens do instrumento. Segundo Reise et al. ${ }^{23}$, sempre que o valor próprio observado for maior do que o seu correspondente na matriz aleatória, concebe-se a existência de um fator. Neste estudo, assumiram-se os parâmetros do banco de dados original (547 participantes e 10 variáveis) com 100 simulações. Os resultados dessa análise podem ser observados na tabela 2.

Tabela 2. Análise paralela para os itens do AUDIT $(n=547)$

\begin{tabular}{lcc}
\hline Valores observados & Valores simulados & Percentis \\
\hline 4,75 & 1,21 & 1,26 \\
1,16 & 1,15 & 1,20 \\
0,86 & 1,10 & 1,13 \\
0,80 & 1,06 & 1,09 \\
0,70 & 1,01 & 1,04 \\
0,50 & 0,97 & 1,00 \\
0,42 & 0,94 & 0,97 \\
0,39 & 0,89 & 0,93 \\
0,23 & 0,85 & 0,89 \\
0,17 & 0,80 & 0,84 \\
\hline
\end{tabular}

Conforme a tabela 2, parece pertinente extrair dois componentes para o conjunto dos 10 itens do AUDIT. Por isso, procedeu-se a uma Análise de Componentes Principais com rotação oblimin, fixando a extração de dois fatores. Os resultados a respeito são apresentados na tabela 3.

Tabela 3. Estrutura componencial do AUDIT

\begin{tabular}{|c|c|c|c|}
\hline \multirow{2}{*}{ Conteúdo dos itens } & \multicolumn{2}{|c|}{ Componente } & \multirow{2}{*}{$h^{2}$} \\
\hline & I & II & \\
\hline $\begin{array}{l}\text { 1. Com que frequência você consome alguma bebida } \\
\text { alcoólica? }\end{array}$ & $0,87^{a}$ & $-0,01$ & 0,76 \\
\hline $\begin{array}{l}\text { 2. Nas ocasiões em que bebe, quantas doses, copos ou } \\
\text { garrafas você costuma tomar? }\end{array}$ & $0,93^{\mathrm{a}}$ & $-0,11$ & 0,78 \\
\hline $\begin{array}{l}\text { 3. Com que frequência você toma "seis ou mais doses" } \\
\text { em uma ocasião? }\end{array}$ & $0,88^{\mathrm{a}}$ & 0,02 & 0,79 \\
\hline $\begin{array}{l}\text { 4. Com que frequência você achou que não seria capaz } \\
\text { de controlar a quantidade de bebida depois de começar? }\end{array}$ & 0,31 & $0,54^{\mathrm{a}}$ & 0,55 \\
\hline $\begin{array}{l}\text { 5. Com que frequência você não conseguiu cumprir com } \\
\text { algum compromisso por causa da bebida? }\end{array}$ & 0,06 & $0,82^{\mathrm{a}}$ & 0,72 \\
\hline $\begin{array}{l}\text { 6. Com que frequência depois de ter bebido muito você } \\
\text { precisou beber pela manhã para se sentir melhor? }\end{array}$ & $-0,11$ & $0,89^{\mathrm{a}}$ & 0,71 \\
\hline $\begin{array}{l}\text { 7. Com que frequência você sentiu culpa ou remorso } \\
\text { depois de beber? }\end{array}$ & $0,61^{\mathrm{a}}$ & 0,06 & 0,41 \\
\hline $\begin{array}{l}\text { 8. Com que frequência você não conseguiu se lembrar do } \\
\text { que aconteceu na noite anterior por causa da bebida? }\end{array}$ & $0,57^{\mathrm{a}}$ & 0,33 & 0,61 \\
\hline $\begin{array}{l}\text { 9. Alguma vez na vida você ou alguma outra pessoa já } \\
\text { se machucou, ou se prejudicou pelo fato de você ter } \\
\text { bebido? }\end{array}$ & $0,41^{\mathrm{a}}$ & 0,20 & 0,29 \\
\hline $\begin{array}{l}\text { 10. Alguma vez na vida algum parente, amigo, médico } \\
\text { ou outro profissional da saúde já se preocupou com você } \\
\text { por causa de bebida ou lhe disse para parar de beber? }\end{array}$ & $0,56^{\mathrm{a}}$ & $-0,07$ & 0,29 \\
\hline Número de itens & 7 & 3 & \\
\hline Valor próprio & 4,75 & 1,16 & \\
\hline \% Variância total explicada & 47,5 & 11,6 & \\
\hline Alfa de Cronbach & 0,84 & 0,69 & \\
\hline
\end{tabular}

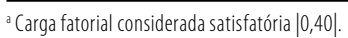

Como se verifica, a estrutura bifatorial dessa medida parece coerente; todos os itens apresentaram saturações consideradas satisfatórias, isto é, igual ou superior a $|0,40|^{24}$. Com base na nomenclatura dos três domínios proposta pela OMS, o primeiro componente pode ser nomeado como Frequência e consequências adversas do consumo, reunindo sete itens (por exemplo, Com que frequência você consome alguma bebida alcoólica? Com que frequência você sentiu culpa ou remorso depois de beber?). Ele apresentou valor próprio de 4,75, explicando 47,5\% da variância total; sua consistência interna (alfa de Cronbach) foi de 0,84. No caso do segundo componente, este foi denominado de Dependência, tendo reunido três itens (por exemplo, Com que frequência você achou que não seria capaz de controlar a quantidade de bebida depois de começar?). Seu valor próprio foi de 1,16, correspondendo à explicação de 11,6\% da variância total, com consistência in- 
terna de 0,69 . Os dois fatores se apresentaram significativamente correlacionados $(r=0,59 ; p \leq 0,00)$.

Finalmente, para complementar as informações acerca da confiabilidade do AUDIT, avaliou-se sua precisão teste-reteste. Para tanto, calculou-se a correlação $r$ de Pearson entre as pontuações totais apresentadas por 49 participantes em dois momentos ( $t_{1}$ e $t_{2}$; tempo de 45 dias entre ambas) Os resultados indicaram correlação forte entre os dados obtidos na primeira e segunda aplicações $\left(r_{t t}=0,94, p<0,01\right)$. Em seguida, compararam-se, por meio de um teste $t$ para amostras emparelhadas, as pontuações médias apresentadas pelos participantes nos dois momentos. Nessa análise não se encontrou diferença significativa entre as médias nos dois tempos $\left(m_{t 1}=0,37, d p=0,49 ; m_{t 2}=0,34, d p=0,47 ; p>\right.$ $0,05)$. Por fim, para assegurar a concordância das pontuações entre as duas aplicações (teste e reteste), procedeu-se, ainda, ao cálculo do coeficiente de correlação intraclasse (ICC; Two-Way Random) para a pontuação total no AUDIT (ICC = 0,96; $p<0,001)$, resultado que corrobora a satisfatória estabilidade temporal da escala.

\section{DISCUSSÃO}

Com base nos resultados, verificou-se que todos os itens do instrumento apresentaram poder discriminativo significativo e correlação item-total acima do mínimo recomendado na literatura $(0,20)^{18}$. Contudo, analisando os valores $t$, percebe-se que, no conjunto, alguns itens são aparentemente mais eficazes para diferenciar os respondentes; possivelmente esse aspecto pode ser justificado devido ao conteúdo dos itens e sua relação com a amostra utilizada neste estudo.

Quanto à fidedignidade do AUDIT, o alfa de Cronbach para o conjunto total de itens foi de 0,86 , o que condiz com a literatura, que aponta como aceitável um alfa maior ou igual a $0,70^{19}$. Coerentemente, a maioria dos estudos sobre confiabilidade do AUDIT apresentou alfas iguais ou superiores a 0,807.

Contudo, é importante destacar as diferenças dos valores dos alfas de Cronbach calculados para cada um dos fatores. Ainda que se reconheça que esse parâmetro apresenta diferenças quando comparados os dois fatores propostos, é necessário considerar, na interpretação dos resultados, o número de itens de cada fator (o fator dependência se apresentou com apenas três itens). Além disso, deve-se ponderar que os itens que abrangem tal fator envolvem aspectos referentes aos sintomas de dependência, características que possivelmente não se aplicam à amostra utilizada nesta pesquisa (estudantes universitários). Em todo caso, cabe assinalar que, para instrumentos de screen e utilizados em estudos exploratórios, alfas próximos a 0,60 já podem ser considerados aceitáveis ${ }^{18,25}$. Quanto à precisão teste-reteste, os resultados também foram favoráveis, na medida em que indicaram estabilidade temporal do AUDIT entre a primeira e a segunda aplicação, assemelhando-se a outros estudos que consideraram períodos de tempos distintos ${ }^{10,13}$.

A estrutura fatorial do AUDIT se apresentou concordante com outros estudos que observaram sua multidimensionalidade ${ }^{6,89,26}$. No presente estudo, considerando os diferentes critérios para extração de fatores propostos na literatura, foi possível estimar a existência de dois fatores, tendo em conta os critérios de Kaiser e Horn. Sobre isso, como aponta a revisão de Meneses-Gaya et al. ${ }^{7}$, tal estrutura é mais comumente encontrada do que a unifatorial, sendo, portanto, um modelo aceitável para a população universitária, uma vez que, segundo Lima et al. ${ }^{9}$, em amostras procedentes de populações com alta prevalência de dependência, os resultados corroboram o modelo unifatorial, mas naquelas em que se observa baixa prevalência de transtornos relacionados com o consumo de álcool, os resultados remetem à estrutura composta por dois ou três fatores.

Acerca da estrutura de dois fatores encontrada, é preciso ponderar que, diferente de outras pesquisas ${ }^{9,8,26}$, a organização dos fatores no presente estudo se deu de maneira distinta. Assim, tendo por base a divisão proposta pela $\mathrm{OMS}^{6}$, o Fator 1 reuniu os itens que se referiram à Frequência (1-3) e às Consequências adversas do consumo (7-10), e o Fator II abrangeu apenas os itens que se relacionavam com a Dependência (4-6).

De maneira geral, como dito, este estudo teve como objetivo avaliar as qualidades psicométricas do AUDIT, conhecendo o poder discriminativo de seus itens, sua consistência interna, precisão teste-reteste e estrutura fatorial. Confia-se que ele tenha sido alcançado satisfatoriamente. No entanto, percebe-se que tais resultados abrangem unicamente informações resultantes de análises exploratórias, o que impossibilita conclusões contundentes acerca da dimensionalidade do instrumento. Diante disso, apontam-se para estudos futuros o emprego de estatísticas mais robustas, como é o caso das modelagens por equação estrutural, e a ampliação da amostra, considerando participantes da população geral e amostras clínicas. Dessa forma, poder-se-ia contrastar os modelos uni e bifatorial.

Além disso, recomenda-se a realização de análises mais específicas, como curvas ROC, que considerem valores de sensibilidade e especificidade, indicando o ponto de corte mais adequado para as amostras utilizadas nesta pesquisa, como se verifica em outros estudos da área ${ }^{9,13}$. Finalmente, ressalta-se a importância de se realizarem estudos de normatização do instrumento, considerando amostras probabilísticas que permitirão a generalização dos resultados e serão úteis na confirmação dos parâmetros psicométricos que têm sido observados neste contexto e em estudos prévios ${ }^{7,13}$.

\section{CONCLUSÃO}

Finalmente, o presente estudo apresenta aspectos importantes sobre a qualidade psicométrica do AUDIT em contex- 
to brasileiro, indicando sua adequação e contribuindo para clarificar possíveis dúvidas acerca da estrutura fatorial dessa medida. Em função dos resultados, torna-se possível considerar o AUDIT como uma medida de triagem parcimoniosa, podendo ser utilizada em diversos campos, como no sistema de saúde, no processo de habilitação de condutores, em empresas e programas clínicos de tratamento.

\section{AGRADECIMENTOS}

O presente estudo compreende parte de um projeto integrado, financiado pelo Conselho Nacional de Desenvolvimento Científico e Tecnológico (Proc. CNPq - 483258/2010-5) e Fundação Cearense de Apoio ao Desenvolvimento Científico e Tecnológico (Proc. FUNCAP - 0033-0052.01.00/10). Os autores agradecem a essas instituições.

\section{REFERÊNCIAS}

1. Barroso T, Mendes A, Barbosa A. Analysis of the alcohol consumption phenomenon among adolescents: study carried out with adolescents in intermediate public education. Rev Lat Am Enfermagem. 2009;17(3):347-53.

2. Mascarenhas MDM, Malta DC, Silva MMA, Carvalho CG, Monteiro RA, Morais Neto OL. Consumo de álcool entre vítimas de acidentes e violências atendidas em serviços de emergência no Brasil, 2006 e 2007. Ciênc Saúde Coletiva. 2009;14(5):1789-96.

3. Andrade AG, Anthony JC. Álcool e suas consequências: uma abordagem multiconceitual. [internet] São Paulo; 2009. Disponível em: http://bvssp.icict.fiocruz.br/lildbi/docsonline/ get.php?id=2047. Acessado em: 1 nov. 2011.

4. Index Psi(BVS-Psi). São Paulo: Biblioteca Virtual em Saúde Psicologia 2011 [internet]. Disponível em: http://www.bvs-psi.org.br/. Acessado em: 12 out. 2011.

5. Google Acadêmico. Scholar Google [internet]. Disponível em: http://scholar.google.com. br/. Acessado em: 12 out. 2011

6. Babor TF, Higgins-Biddle J, Saunders J, Monteiro M. The alcohol use disorders identification test: guidelines for use in primary care. [internet] 2. ed. Genebra: World Health Organization; 2001. Disponível em: http://whqlibdoc.who.int/hq/2001/who_msd_msb_01.6a. pdf. Acessado em: 1 nov. 2011.

7. Meneses-Gaya C, Zuardi AW, Loureiro SR, Crippa JAS. Alcohol Use Disorders Identification Test (AUDIT): an updated systematic review of psychometric properties. Psychol Neurosci. 2009;2(1):83-97.
8. Rist F, Glöckner-Rist A, Demmel R. The Alcohol Use Disorders Identification Test revisited: establishing its structure using nonlinear factor analysis and identifying subgroups of respondents using latent class factor analysis. Drug Alcohol Depend. 2009;100(1-2):71-82.

9. Lima CT, Freire ACC, Silva APB, Teixeira R, Farrell M, Prince M. Concurrent and construct validity of the AUDIT in an urban Brazilian sample. Alcohol Alcohol. 2005;40(6):584-89.

10. Selin $\mathrm{KH}$. Test-retest reliability of the alcohol use disorder identification test in a general population sample. Alcohol Clin Exp Res. 2003;27(9):1428-35.

11. Martins RA, Manzatto AJ, Cruz LN, Poiate SMG, Scarin ACCF. Utilização do Alcohol Use Disorders Identification Test (AUDIT) para identificação do consumo de álcool entre estudantes do ensino médio. Interam J Psychol. 2008;42(2):307-16.

12. Brasil Ministério da Saúde. Rastreamento: Cadernos de Atenção Primária. [internet]. Brasília: Secretaria de Atenção à Saúde; 2010. Disponível em: http://189.28.128.100/dab/docs/ publicacoes/cadernos_ab/abcad29.pdf. Acessado em: 1 nov. 2011.

13. Moretti-Pires RO, Corradi-Webster CM. Adaptation and validation of the Alcohol Use Disorders Identification Test (AUDIT) for a river population in the Brazilian Amazon. Cad Saude Publica. 2011;27(3):497-509.

14. Méndez B. Uma versão brasileira do AUDIT - Alcohol Use Disorders Identification Test [dissertação]. Pelotas: Universidade Federal de Pelotas, Faculdade de Medicina, Departamento de Medicina Social; 1999.

15. Paquali L. Instrumentação psicológica: fundamentos e práticas. Porto Alegre: Artmed; 2010.

16. Saunders JB, Aasland OG, Babor TF, Fuente JR, Grant M. Development of the Alcohol Use Disorders Identification Test (AUDIT): WHO Collaborative Project on Early Detection of Persons with Harmful Alcohol Consumption-II. Addiction. 1993;88(6):791-804.

17. Tabachnick BG, Fidell LS. Using multivariate statistics. 5. ed. Boston: Allyn \& Bacon; 2006

18. Clark LA, Watson D. Constructing validity: basic issues in objective scale development. Psychol Assess. 1995;7(3):309-19.

19. Nunnally JC, Bernstein IH, Arellano JAV, Guillén MT. Teoría psicométrica. México: Mcgraw-hill; 1995.

20. Fava JL, Velicer WF. The effects of underextraction in factor and component analyses. Educ Psychol Meas. 1996;56(6):907-29.

21. Laros J, Puente-Palacios K. Validação cruzada de uma escala de clima organizacional. Estudos de Psicologia. 2004;9(1):113-9.

22. Hayton JC, Allen DG, Scarpello V. Factor retention decisions in exploratory factor analysis: a tutorial on parallel analysis. Organizational Research Methods. 2004;7(2):191.

23. Reise SP, Waller NG, Comrey AL. Factor analysis and scale revision. Psychol Assess. 2000;12(3):287-97.

24. Hair JF, Black WC, Babin BJ, Anderson RE, Tatham RL. Multivariate data analysis. 5. ed. New Jersey: Prentice hall; 1998

25. Garson GD. Scales and standard measures. North Carolina State University; 2007. Disponível em: http://faculty.chass.ncsu.edu/garson/PA765/standard.htm. Acessado em: 1 nov. 2011.

26. Von-der-Pahlen B, Santtila P, Witting K, Varjonen M, Jern P, Johansson A, et al. Factor structure of the Alcohol Use Disorders Identification Test (AUDIT) for men and women in different age groups. J Stud Alcohol Drugs. 2008;69(4):616-21. 